\title{
Pulsed Electromagnetic Fields Increase Pigmentation through the p-ERK/p-p38 Pathway in Zebrafish (Danio rerio)
}

\author{
Yu-Mi Kim ${ }^{1}$, Han-Moi Lim ${ }^{1}$, Hyang-Seon $\mathrm{Ro}^{2}{ }^{2}$, Ga-Eun $\mathrm{Ki}^{1}$ and Young-Kwon Seo ${ }^{1, *(D)}$ \\ 1 Department of Medical Biotechnology (BK21 Plus Team), Dongguk University, Goyang-si 10326, Korea; \\ kjmtik@nate.com (Y.-M.K.); gksahl321@gmail.com (H.-M.L.); gigaeun1104@gmail.com (G.-E.K.) \\ 2 R\&D Center, NOWCOS, Seoul 08506, Korea; rohs@nowcos.co.kr \\ * Correspondence: bioseo@dongguk.edu; Tel: +82-31-961-5154; Fax: +82-31-961-5621
}

\begin{abstract}
Melanogenesis is a biological process resulting in the production of melanin pigment, which plays an important role in the prevention of sun-induced skin injury, and determines hair and skin color. So, a wide variety of approaches have been proposed to increase the synthesis of melanin. This study evaluated the effects of pulsed electromagnetic fields (PEMFs) on the pigmentation of zebrafish (Danio rerio) in vivo. We stimulated pigmentation in zebrafish by using specific frequencies and intensities of PEMFs. This study focuses on pigmentation using PEMFs, and finds that PEMFs, at an optimal intensity and frequency, upregulate pigmentation by the stimulated expression of tyrosinase-related protein 1 (TRP1), dopachrome tautomerase (DCT) through extracellular signal-regulated kinase(ERK) phosphorylation, and p38 phosphorylation signaling pathways in zebrafish. These results suggest that PEMFs, at an optimal intensity and frequency, are a useful tool in treating gray hair, with reduced melanin synthesis in the hair shaft or hypopigmentation-related skin disorders.
\end{abstract}

Keywords: pigmentation; melanogenesis; electromagnetic field; zebrafish; Danio rerio; vitiligo

\section{Introduction}

Melanin is a unique pigmented biopolymer synthesized by specialized cells known as melanocytes, which are dendritic cells that exist in relatively minor populations in the skin, hair, eyes, and other locations [1]. The natural tanning process occurs as a response to exposure to UV radiation. [2]. Several recent studies have focused on the treatment of diseases caused as a result of hypopigmentation of the skin or hair. Vitiligo is a common depigmenting skin disorder, with a worldwide prevalence of $0.5-1 \%$ [3]. A variety of physical treatments are used to induce melanin production in the melanocytes of vitiligo patients, who are characterized by a partial loss of melanocytes from the epidermis of the skin. Recently, a lot of alternative therapies have been proposed as vitiligo treatments. Narrow-band UVB radiation has been shown to be effective in the treatment of vitiligo [4]. In addition, a variety of other approaches for inducing pigmentation have been proposed, including the use of an excimer laser $(308 \mathrm{~nm})$ [5,6], monochromatic emission at $355 \mathrm{~nm}$ (UVA1) laser [7], helium-neon laser [8], neodymium-doped yttrium aluminum garnet(Nd: YAG) laser [9], and Q-switched ruby laser $[10,11]$. Safe methods to promote melanogenesis would be valuable in medicine and cosmetics.

The use of pulsed electromagnetic fields (PEMFs) has recently been explored as an effective method because of its non-invasiveness, safety, lack of side-effects, convenience, and superior treatment prospects in the treatment of several refractory bone diseases, such as non-unions and the delayed 
healing of fractures [12]. Furthermore, several studies have suggested that electromagnetic fields (EMFs) are effective in cartilage repair [13], in reducing diabetic neuropathic pain [14], in adult hippocampal neurogenesis [15], in angiogenesis [16], and in skin wound repair [17]. Our previous studies have shown that PEMF induces melanogenesis in B16F10 melanoma and human melanocyte cells [18-20]. Pigmentation is a complex process involving many factors. Microphthalmia-associated transcription factor (MITF) is the master gene involved in pigmentation, and it controls several crucial mechanisms in melanocytes, such as melanogenesis, dendricity, and proliferation, in response to environmental factors [21]. The activity of melanogenic enzymes and melanosome transfer can affect pigmentation. Tyrosinase, tyrosinase-related protein 1 (TRP 1), and TRP 2 are the melanocyte-specific enzymes involved in melanin biosynthesis [22]. Recently, it was reported that melanogenesis is also controlled by various intracellular signaling molecules, such as mitogen-activated protein kinases (MAPK) [23].

The zebrafish (Danio rerio) has become a favored model for biochemical studies because it is an efficient and robust alternative to conventional animal experiments [24]. It is a small tropical freshwater fish, and is a useful vertebrate model organism because of its small size, large clutches, transparent body, and physiological similarity to mammals, in addition to its low cost of breeding [25]. In addition, zebrafish have melanin pigmentation on their body surface and rapidly alter their pigmentation in response to environmental changes, which enables a facile investigation of pigmentation without the need for complicated experimental procedures [26].Therefore, the zebrafish is an ideal model for the study of melanogenesis in the context of pigmentation. In the present study, we applied PEMFs for the stimulation of pigmentation in zebrafish. Zebrafish larvae were stimulated at intensities of 2,4 , and $20 \mathrm{G}$, at a constant frequency of $60 \mathrm{~Hz}$. In order to verify the changes in pigmentation, we performed melanin assays, a Western blot analysis, and immunohistochemical staining. Specifically, we analyzed the changes in ERK and p38 signaling associated with MITF regulation, due to the PEMFs.

\section{Results}

\subsection{Melanin Assay}

To test whether the PEMFs induced pigmentation in zebrafish, the amount of melanin was determined using a melanin content assay after exposure to PEMFs. The melanin content of the PEMF-treated zebrafish larvae was more than that of the untreated zebrafish larvae (Figure 1B). The $2 \mathrm{G}$ group showed an increase of 1.22-fold, the $4 \mathrm{G}$ group of $\sim 1.32$-fold, and the 20 G group showed an approximately 1.16-fold increase over the negative control group. The larvae exposed to $4 \mathrm{G}$ exhibited a particularly large increase relative to the controls. These results suggest that the PEMFs, especially at $4 \mathrm{G}$, increased pigmentation in zebrafish.

\subsection{RT-PCR}

The mRNA expression levels of the key melanogenesis-related genes, dct, tyrp1, mitfa, and mc1r, were measured at $5 \mathrm{dpf}$ (days post-fertilization), as shown in Figure 1. The Dct mRNA levels in the zebrafish exposed to PEMFs were on average 1.4-fold higher than those in the control. The zebrafish exposed to PEMFs at $4 \mathrm{G}$ showed a dct expression $\sim 2.0$ times higher than that in the controls. The mitfa expression levels increased at all intensities of the PEMFs. The mitfa mRNA levels in the zebrafish exposed to 4 G PEMFs were 2.0 times higher than those in the controls. The zebrafish exposed to $4 \mathrm{G}$ showed elevated $m c 1 r$ expressions, 1.4 times higher than that in the control. Thus, the PEMF exposure was found to stimulate the expression of melanogenesis-related mRNA.

\subsection{Western Blotting}

Based on the results of the RT-PCR, the expression of the pigmentation-related proteins was measured using Western blot analysis after exposure to 4 G PEMFs at $15 \mathrm{dpf}$. As shown in Figure 1E, relative to the controls, the expression of all pigmentation-related proteins increased in zebrafish 
exposed to PEMFs. To investigate the postulated signaling mechanism involved in the effect of PEMFs on pigmentation in zebrafish, zebrafish embryos were treated with PEMFs. In order to study the processes related to pigmentation in the zebrafish exposed to PEMF, we assessed the activation of p-ERK and p-p38 signaling. The protein expression of ERK and p-ERK were determined using Western blotting. Treatment with PEMF led to a significant decrease in phosphorylated-ERK. Conversely, the p-p38 activation was increased in the cells exposed to PEMFs. Thus, the PEMF exposure stimulated melanogenesis in zebrafish.

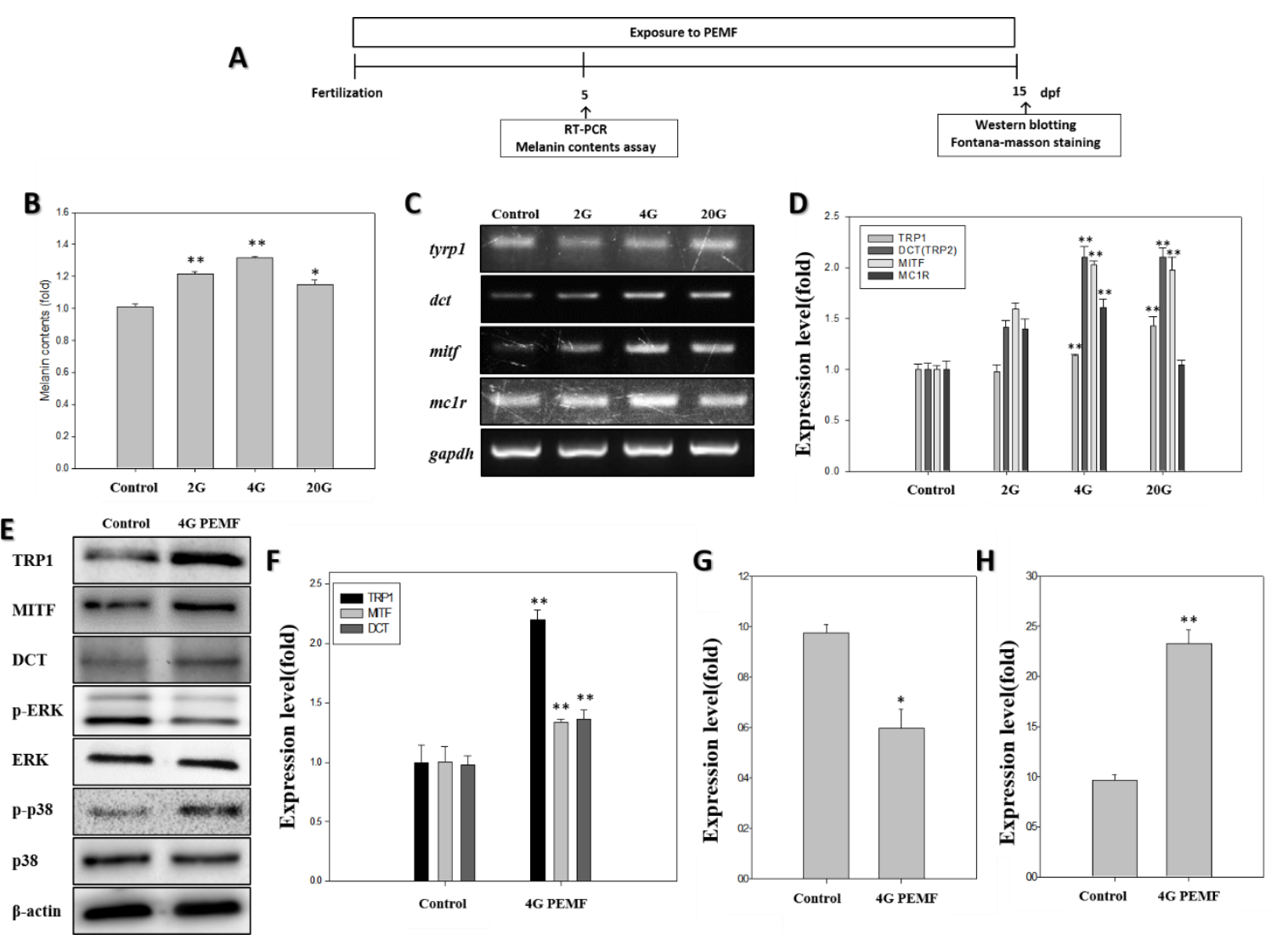

Figure 1. Effect of pulsed electromagnetic fields (PEMFs) on the pigmentation of zebrafish after exposure to PEMFs. (A) Schematic representation of the schedule of the zebrafish pigmentation study. After PEMF exposure, the melanin content was determined. (B) The melanin content in zebrafish $(\mathrm{n}=80)$ was detected using the melanin content assay at $5 \mathrm{dpf}$. (C) The mRNA expression, detected using reverse transcription polymerase chain reaction, results in zebrafish $(\mathrm{n}=20)$ after exposure to PEMFs at $5 \mathrm{dpf}$. (D) The mRNA expression of melanogenesis-related genes, using glyceraldehyde-3-phosphate dehydrogenase (GAPDH) as the reference gene. The effect of PEMF on the protein levels of tyrosinase-related protein-1 (TRP1), microphthalmia-associated transcription factor (MITF), dopachrome tautomerase (DCT), extracellular signal-regulated kinase (ERK), p-ERK, p-p38, p38, and b-actin in zebrafish $(n=30)$ at $15 \mathrm{dpf}$. (E) The Western blotting analysis of the pigmentation-related proteins; (F) TRP1, MITF, and DCT expression level; (G) p-ERK expression; (H) p-p38 expression. Each bar represents the mean \pm standard error of the independent experiments performed in triplicate $(\mathrm{n}=5) .{ }^{*} p<0.05,{ }^{* *} p<0.01$, compared to the control.

\subsection{Pigmentation}

A region of interest was selected to measure the pigmentation area, indicated by a white outline embracing the dorsal pigment spot from midway between the eyes, around the pigmented eyes, to the base of the head (Figure 2A). The density of the pigmented area in the treated embryos was normalized to that in the control embryos using ImageJ software. The treatment of the zebrafish 
embryos with PEMFs for $15 \mathrm{dpf}$ significantly increased the skin melanin formation in the developing larvae (Figure 2B).
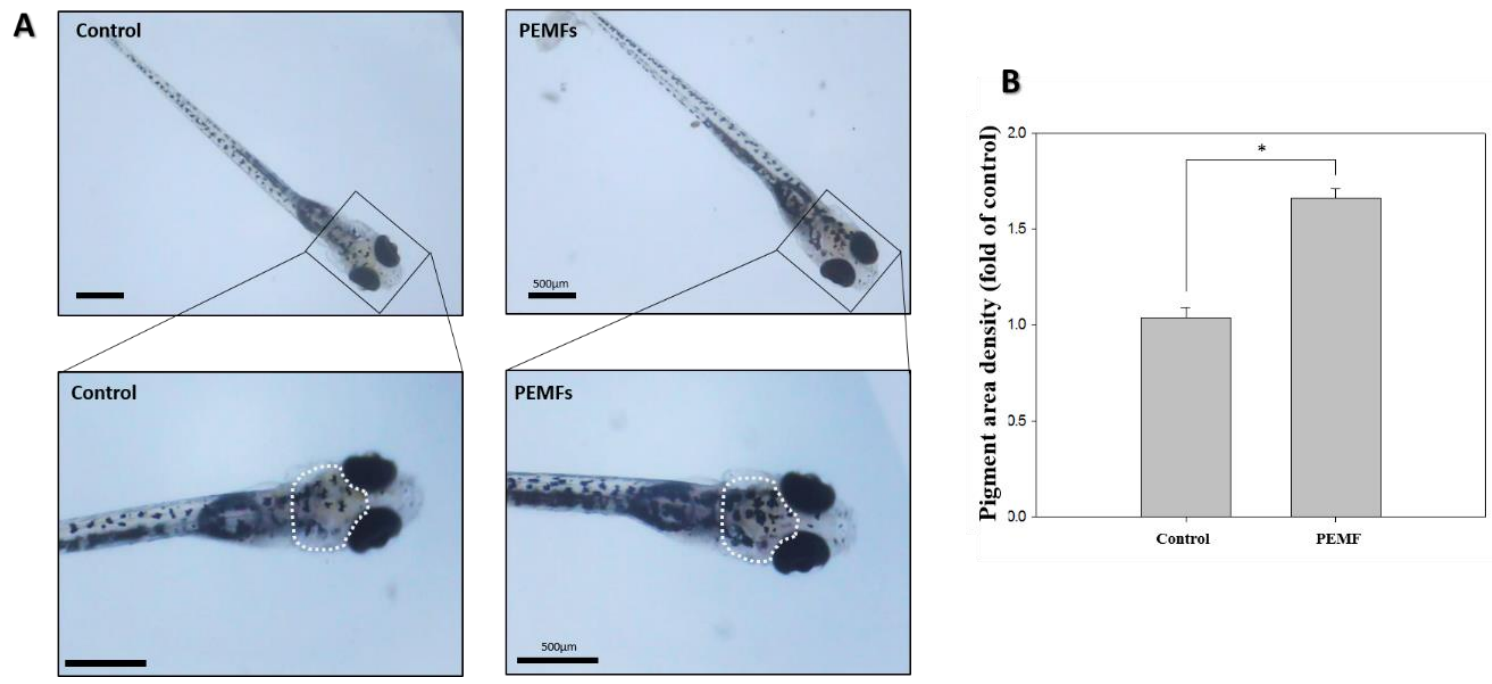

Figure 2. Effects of 4 G PEMF on pigmentation in zebrafish. (A) Synchronized embryos $(n=20)$ were exposed to the PEMF at the indicated intensity and frequency. The effects on zebrafish pigmentation were observed under a stereomicroscope via inferior views of the embryos at $15 \mathrm{dpf}$. (B) The pigmentation area density in the treated embryos, indicated by a white outline, was normalized to that of the control embryos using the ImageJ software. ${ }^{*} p<0.05$, compared to the control. The scale bar is $500 \mu \mathrm{m}$.

\subsection{Fontana-Masson Staining}

To visualize the melanin, the zebrafish larvae were stained using Fontana-Masson staining, which is used to confirm melanin synthesis. A region of interest was selected to measure the pigmentation area, indicated by a white outline. As shown in Figure 3, compared with the controls, the amount of melanin granules was significantly increased due to PEMF exposure (black color).
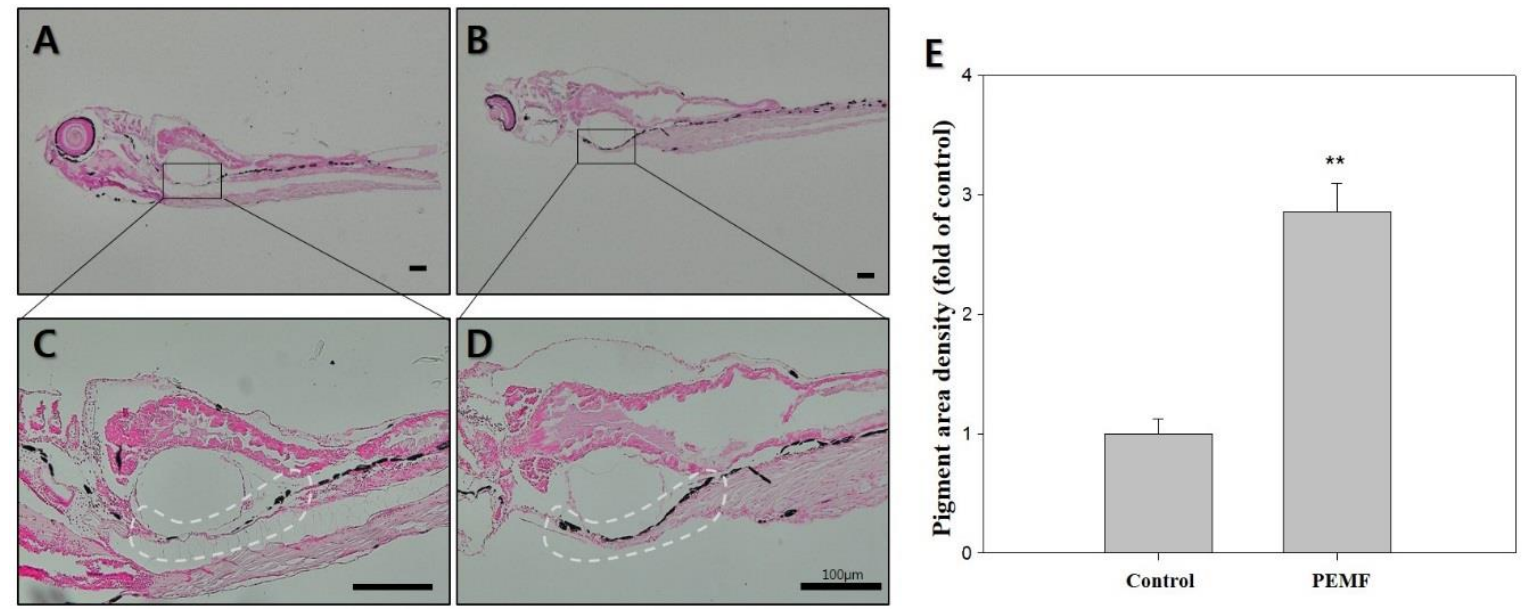

Figure 3. Effects of $4 \mathrm{G}$ PEMF in zebrafish at $15 \mathrm{dpf}$. Representative images of Fontana-Masson-stained zebrafish (dark color indicates secreted melanin). (A,C) control, (B,D) EMF. (E) the pigmented area density in figure (A-D) was normalized to that of the control, using ImageJ sotware. ${ }^{* *} p<0.05$, compared to the control. Original magnification: $(\mathbf{A}, \mathbf{B}) \times 40 ;(\mathbf{C}, \mathbf{D}) \times 100$ scale bar; $100 \mu \mathrm{m}$. 


\section{Discussion}

A wide variety of approaches have been proposed to increase the synthesis of melanin, including the use of an excimer laser $(308 \mathrm{~nm})$ [5,6], UVA1 laser [7], helium-neon laser [8], $\mathrm{Nd}$ : YAG laser [9], and Q-switched ruby laser [10,11]. The electric appliances that have magnetic fields in domestic use are easily visible around us, such as microwave cookers $(0.0037+/-0.0014 \mathrm{G})$ and washing machines $(0.0027+/-0.0014 \mathrm{G})$ [27]. On the one hand, electromagnetic fields (EMFs) are abundantly present in modern life, and interest regarding the effect of extremely low frequency (ELF) EMF on human health has been increasing. Different types of magnetic and electromagnetic fields are now used in medical fields, such as in diagnostics (e.g., magnetic resonance, scanning, and microwave imaging) and therapy [28]. Extremely low-frequency electromagnetic fields (ELF-EMF) influence cell differentiation [29,30], cell proliferation, mitochondrial activity [31], cell survival [32], regulate the cell cycle [33], and neurogenesis [15]. Nevertheless, there is a lack of studies examining general EMF effects on melanogenesis. To address this, in a previous study, we evaluated the effect of PEMFs on melanin synthesis in vitro. We found that PEMFs promoted melanogenesis in B16F10 melanoma cells and human melanocyte cells. We used RT-PCR and Western blotting to show that PEMFs stimulate the expression of melanogenesis-related genes and proteins. Thus, our previous findings suggested that the PEMF stimulation significantly promoted melanogenesis [18-20].

Zebrafish have become a useful model for biochemical studies [24], and have been established as a new in vivo model for evaluating the pigmentation activity of melanogenic regulatory compounds [34]. In the present study, we applied PEMFs for the stimulation of pigmentation in zebrafish. To investigate the hyperpigmentation effect of PEMFs, a melanin assay was used. As shown in Figure 1B, the melanin content increased when the zebrafish were exposed to PEMF. Treatment at $4 \mathrm{G}$ strongly increased the melanin content, compared with the control group. Thus, PEMFs were found to promote pigmentation in zebrafish. The pigmentation in the treated embryos was observed under a stereomicroscope at $5 \mathrm{dpf}$ (Figure 2A), and the density of the pigmentation area was normalized to that in the control embryos. The pigmentation area in the untreated zebrafish was clearly less than that in the PEMF treated-zebrafish larvae (Figure 2B). These results are consistent with those reported in our previous in vitro studies [18-20].

Pigmentation in zebrafish embryos is due to the activity of some major enzymes, including tyrp1, $d c t$, and mitfa. Mitf is the master gene in pigmentation and controls several key mechanisms, such as pigmentation in response to environmental factors, including UV radiation [35]. The activation of MITF induces the expression of the key enzymes of pigmentation, dct and tyrp1, leading to the production of melanin. In addition, $m c 1 r$ is a key regulator of melanosome dispersal in zebrafish [36].

The expression of these enzymes was determined using RT-PCR, and the results showed that treatment with $4 \mathrm{G}$ EMF induced the expression of mitf and dct (Figure 1D). Moreover, the dct, tyrp1, and mitf expressions, as quantified by Western blotting, increased in the PEMF-treated group. Western blotting using anti-DCT antibody indicated an increase in the expression of these melanophore-specific proteins [37]. Previous studies have demonstrated that MAPK kinases, including ERK and p38, play an important role in pigmentation [38]. It is well established that the ERK signaling pathway is involved in cell proliferation and differentiation; furthermore, the ERK signaling pathway has been identified as a negative regulator of melanogenesis [39]. The upregulation of phosphorylated ERK signaling is related to the reduction of melanin synthesis [20]. However, the phosphorylation of p38 MAPKs can upregulate MITF expression [34,40]. The mitogen-activated protein kinase (MAPK) cascades are an important set of signaling pathways activated in response to EMF in most systems [41]. Thus, to clarify the signaling pathway involved in the PEMF action on melanin synthesis in zebrafish, we examined the phosphorylation of ERK and p38 (Figure 1G,H). The results showed a significant decrease in the phosphorylation of ERK in the PEMF-treated group, which can also lead to the stimulation of the melanogenic pathway by accelerating the MITF activation (Figure 1E,F). The results indicate that the treatment of PEMFs at an optimal intensity and frequency influence the mechanically sensitive kinases, such as ERK and p38. To visualize melanin, the samples were stained with 
Fontana-Masson staining, and the pigmented area density was determined (Figure 3). Silver nitrate $\left(\mathrm{AgNO}_{3}\right)$ reacts with melanin to produce metallic silver $(\mathrm{Ag})$, resulting in a black stain that can be visualized using a light microscope. As shown in Figure 3, compared with the controls, the amount of melanin granules was significantly increased due to PEMF exposure (dark brown color). The results demonstrate that PEMFs at a specific frequency can stimulate pigmentation in zebrafish. These results may indicate that the optimal condition of PEMFs is good tool for hyperpigmentation therapy for gray hair treatment when melanogenesis is reduced in the hair-follicle, or for hipopigmentation-related skin disorders. As the embryos have been exposed to PEMFs right after birth, more research should be performed to determine whether their effect was a developmental defect.

\section{Materials and Methods}

\subsection{Zebrafish Maintenance and Embryo Collection}

Following standard procedures, adult zebrafish were kept in a circulating filtration system $\left(28 \pm 0.5^{\circ} \mathrm{C}, 14: 10 \mathrm{~h}\right.$ light:dark) and were fed three times a day. The male and female zebrafish were separated until mating and spawning. After natural spawning, the fertilized embryos were collected and used for the experiments. The embryos were placed individually in each well on 96-well plate filled with $100 \mu \mathrm{L}$ of water containing sea salt. The embryo and larval developmental stages were expressed in the days post-fertilization (dpf) [42].

\subsection{PEMFs Exposure}

We used a Helmholtz coil that was able to generate PEMFs; the apparatus is depicted in Figure 4. The stimulus frequency was $60 \mathrm{~Hz}$ and teh stimulus wave was in a pulse form. The electromagnetic field device was placed in a $28.5^{\circ} \mathrm{C}$ incubator. The unit of measurement of the magnetic density is gauss $(G)$ and the zebrafish embryos were stimulated with PEMFs at intensities of 2, 4, and $20 \mathrm{G}$ for 5 or 15 days. The control group was placed in a separate incubator so as to avoid PEMF exposure.
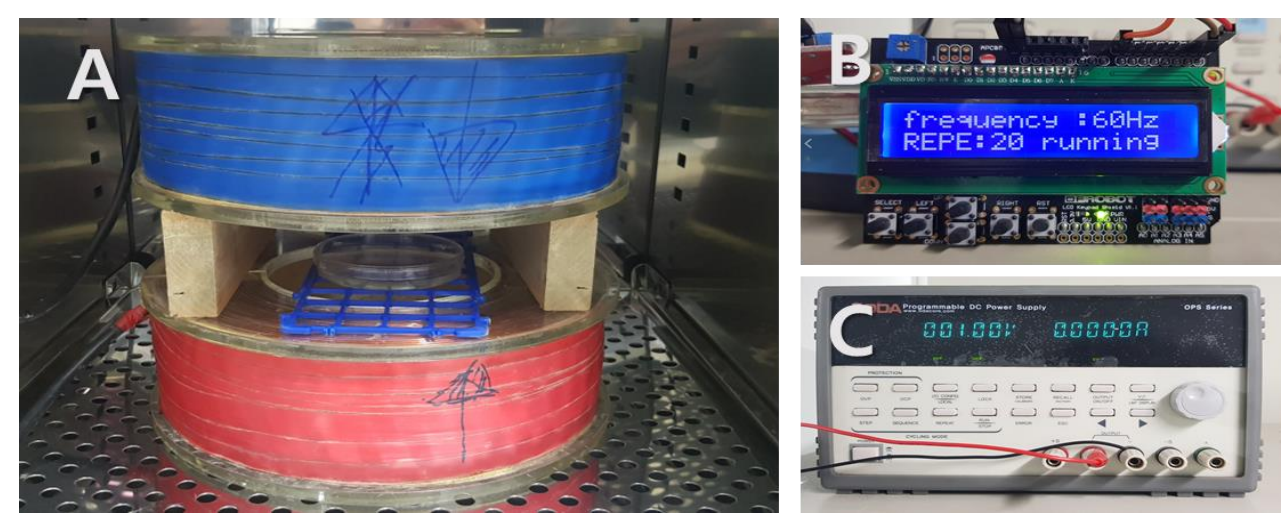

Figure 4. Photograph of the electromagnetic field (EMF) device in incubator. Pulsed EMF was generated using a pair of Helmholtz coils. (A) Helmholtz coil; (B) function generator; (C) power supply.

\subsection{Microscopy}

The embryos from the wild-type control group or from the PEMF-exposed groups were observed and photographed under light microscopy so as to examine the pigmentation.

\subsection{Melanin Assay}

The compound treatment and phenotype-based evaluation were done as previously described [43]. For examining the melanin content, extracts were prepared from $5 \mathrm{dpf}$ zebrafish larvae. Simply put, the embryos were collected and were dissolved in a lysis buffer. After centrifugation, the melanin was extracted into $1 \mathrm{~N} \mathrm{NaOH}$, and was incubated at $100{ }^{\circ} \mathrm{C}$ for $10 \mathrm{~min}$. The optical density of the 
supernatant was measured at $405 \mathrm{~nm}$. The results of the treated embryos were compared to those of the appropriate controls, and the final measurements were reported as percentages of the control sample measurements.

\subsection{Reverse Transcriptase-PCR Analysis}

RT-PCR was used to detect the pigmentation-related genes expressed in the $5 \mathrm{dpf}$ embryos. The total RNA in the zebrafish embryos was extracted using the TRIzol Reagent (Ambion, Austin, TX, USA). The reverse transcriptase reactions were used to synthesize the cDNA from $1 \mu \mathrm{g}$ of total RNA, using an advantage RT-for-PCR kit (Clontech, Palo Alto, CA, USA). The reverse transcriptase-PCR analysis was performed according to the manufacturer's instructions. The expression of the genes encoding tyrosinase-related protein 1a (tyrp1a), dopachrome tautomerase (dct or tyrp2), microphthalmia-associated transcription factor a (Mitfa or nacre), melanocortin 1 receptor (mc1r), and gapdh (glyceraldehyde 3-phosphate dehydrogenase) were evaluated. The bands were visualized using Molecular Imager ChemiDoc XRS+ (Bio-Rad, Hercules, CA, USA). The software Image J (National Institutes of Health, Bethesda, MD, USA) was used for the quantitative analysis of the results.

\subsection{Western Blotting}

The zebrafish embryos were lysed using a buffer containing $2 \%$ SDS, $0.1 \mathrm{mg} / \mathrm{mL}$ bromophenol blue in Tris- $\mathrm{HCl}\left(\mathrm{pH}\right.$ 6.8), 5\% 2-mercaptoethanol, and 10\% glycerol, and were boiled at $100{ }^{\circ} \mathrm{C}$ for $10 \mathrm{~min}$. The protein was quantified using Bicinchoninic acid (23225, Thermo fisher Scientific, Waltham, MA, USA). Subsequently, the sample was subjected to sodium dodecyl sulphate-polyacrylamide gel electrophoresis, and the protein was transferred from the gel to a nitrocellulose membrane. Anti-DCT (Thermo Fisher Scientific, Waltham, MA, USA), anti-MITF (Origene Technologies, Zug, Switzerland), anti-TRP1 (Abcam, Cambridge, UK), anti-ERK (Cell Signaling Technology, Danvers, MA, USA), anti-p-ERK (Cell Signaling Technology, Danvers, MA, USA), anti-p38 (Antibodies-Online, Aachen, Germany), anti-p-p38 (Thermo Fisher Scientific, Waltham, MA, USA), and anti- $\beta$-Actin (Abcam, Cambridge, UK) antibodies were used as the primary antibodies in Western blotting. The blots were incubated with the primary antibodies, and were then further incubated with a horseradish peroxidase-conjugated secondary antibody. The chemiluminescent protein bands were photographed using Molecular Imager ChemiDoc XRS (Bio-Rad, Hercules, CA, USA). To analyze and quantify the Western blotting image, Image J software (National Institutes of Health, Bethesda, MD, USA) was used.

\subsection{Fontana-Masson Silver Staining}

We performed a densitometric analysis to visualize melanin in zebrafish. Fontana-Masson silver staining was performed using a previously described method [44]. Formalin-fixed and paraffin sections were processed on slides and stained with silver nitrate (Kojima Chemical, Kashiwabara, Japan) for $1 \mathrm{~h}$ at $56^{\circ} \mathrm{C}$ and washed with distilled water. Then, the slides were fixed in $5 \%$ sodium thiosulfate solution (Duksan, Seoul, Korea) for $5 \mathrm{~min}$, and were washed with distilled water. After that, they were stained with nuclear fast red solution (Sigma Aldrich, Saint Louis, MO, USA) for $5 \mathrm{~min}$, and were washed with distilled water three times. Finally, after dehydration with $95 \%$ ethanol and $100 \%$ ethanol, the slides were washed two times with xylene (Duksan, Seoul, Korea).

\subsection{Statistical Analysis}

The data were analyzed using one-way analysis of variance (ANOVA) and Student's $t$-test. When the value of $p$ was $<0.05$ or $<0.01$, the difference between the means was considered significant $\left({ }^{*} p<0.05,{ }^{* *} p<0.01\right)$. Graphical representations were produced using the Sigmaplot 2001 software. 


\section{Conclusions}

In the present study, we investigated the pro-pigmentation effect of PEMFs in a zebrafish model. Our data suggest that PEMFs promote pigmentation by inducing MITF and DCT, which are mediated through a reduction of ERK phosphorylation and an upregulation of p38 phosphorylation. These results suggest that PEMFs, at an optimal intensity and frequency, are a useful tool for treating gray hair with reduced melanin synthesis in the hair shaft, or hypopigmentation-related skin disorders such as vitiligo.

Author Contributions: Y.-M.K. conceived and designed experiments. Y.-M.K., H.-M.L., H.-S.R. and G.-E.K. performed experiment and analyzed the data. Y.-M.K. wrote the paper. Y.-K.S. was responsible for overall study design and supervised the project.

Funding: This study was supported by a grant of the Korean Health Technology R\&D Project, Ministry of Health \& Welfare, Republic of Korea (HN14C0086).

Conflicts of Interest: The authors declare no conflict of interest.

\section{References}

1. Jung, G.D.; Yang, J.Y.; Song, E.S.; Par, J.W. Stimulation of melanogenesis by glycyrrhizin in B16 melanoma cells. Exp. Mol. Med. 2001, 33, 131-135. [CrossRef] [PubMed]

2. Yang, J.Y.; Koo, J.H.; Song, Y.G.; Kwon, K.B.; Lee, J.H.; Sohn, H.S.; Park, B.H.; Jhee, E.C.; Park, J.W. Stimulation of melanogenesis by scoparone in B16 melanoma cells. Acta Pharmacol. Sin. 2006, 27, 1467-1473. [CrossRef] [PubMed]

3. Lommerts, J.E.; Uitentuis, S.E.; Bekkenk, M.W.; de Rie, M.A.; Wolkerstorfer, A. The role of phototherapy in the surgical treatment of vitiligo: A systematic review. J. Eur. Acad. Dermatol. Venereol. JEADV 2018, 32, 1427-1435. [CrossRef] [PubMed]

4. Hartmann, A.; Lurz, C.; Hamm, H.; Brocker, E.B.; Hofmann, U.B. Narrow-band UVB311 nm vs. broad-band UVB therapy in combination with topical calcipotriol vs. placebo in vitiligo. Int. J. Dermatol. 2005, 44, 736-742. [CrossRef] [PubMed]

5. Fritz, K.; Salavastru, C. [The $308 \mathrm{~nm}$ Excimer laser:Treatment of vitiligo and hypopigmentation]. Der Hautarzt Zeitschrift fur Dermatologie Venerologie und verwandte Gebiete 2018, 69, 44-47. [CrossRef] [PubMed]

6. Shin, S.; Hann, S.K.; Oh, S.H. Combination treatment with excimer laser and narrowband UVB light in vitiligo patients. Photodermatol. Photoimmunol. Photomed. 2016, 32, 28-33. [CrossRef] [PubMed]

7. Lotti, T.; Franca, K. UVA 1 laser in the treatment of patients with vitiligo. Dermatol. Ther. 2017, 30. [CrossRef] [PubMed]

8. Byun, J.W.; Babitha, S.; Kim, E.K.; Shin, J. A successful helium-neon laser and topical tacrolimus combination therapy in one child with vitiligo. Dermatol. Ther. 2015, 28, 333-335. [CrossRef] [PubMed]

9. Majid, I.; Imran, S. Depigmentation Therapy with Q-Switched Nd: YAG Laser in Universal Vitiligo. J. Cutan. Aesthet. Surg. 2013, 6, 93-96. [CrossRef] [PubMed]

10. Njoo, M.D.; Vodegel, R.M.; Westerhof, W. Depigmentation therapy in vitiligo universalis with topical 4-methoxyphenol and the Q-switched ruby laser. J. Am. Acad. Dermatol. 2000, 42, 760-769. [CrossRef] [PubMed]

11. Kim, Y.J.; Chung, B.S.; Choi, K.C. Depigmentation therapy with Q-switched ruby laser after tanning in vitiligo universalis. Dermatol. Surg. 2001, 27, 969-970. [PubMed]

12. Yuan, J.; Xin, F.; Jiang, W. Underlying Signaling Pathways and Therapeutic Applications of Pulsed Electromagnetic Fields in Bone Repair. Cell. Physiol. Biochem. 2018, 46, 1581-1594. [CrossRef] [PubMed]

13. Benazzo, F.; Cadossi, M.; Cavani, F.; Fini, M.; Giavaresi, G.; Setti, S.; Cadossi, R.; Giardino, R. Cartilage repair with osteochondral autografts in sheep: Effect of biophysical stimulation with pulsed electromagnetic fields. J. Orthop. Res. 2008, 26, 631-642. [CrossRef] [PubMed]

14. Weintraub, M.I.; Herrmann, D.N.; Smith, A.G.; Backonja, M.M.; Cole, S.P. Pulsed electromagnetic fields to reduce diabetic neuropathic pain and stimulate neuronal repair: A randomized controlled trial. Arch. Phys. Med. Rehab. 2009, 90, 1102-1109. [CrossRef] [PubMed] 
15. Leone, L.; Fusco, S.; Mastrodonato, A.; Piacentini, R.; Barbati, S.A.; Zaffina, S.; Pani, G.; Podda, M.V.; Grassi, C. Epigenetic modulation of adult hippocampal neurogenesis by extremely low-frequency electromagnetic fields. Mol. Neurobiol. 2014, 49, 1472-1486. [CrossRef] [PubMed]

16. Greenough, C.G. The effects of pulsed electromagnetic fields on blood vessel growth in the rabbit ear chamber. J. Orthop. Res. 1992, 10, 256-262. [CrossRef] [PubMed]

17. Romero-Sierra, C.; Halter, S.; Tanner, J.A.; Roomi, M.W.; Crabtree, D. Electromagnetic fields and skin wound repair. J. Microw. Power 1975, 10, 59-70. [CrossRef] [PubMed]

18. Cho, S.-E.; Kim, Y.-M.; Kang, K.-H.; Kim, S.-C.; Park, J.-K.; Seo, Y.-K. Pigmentation effect of electromagnetic fields at various intensities to melanocytes. Tissue Eng. Regen. Med. 2016, 13, 560-567. [CrossRef]

19. Kim, Y.M.; Cho, S.E.; Kim, S.C.; Jang, H.J.; Seo, Y.K. Effects of Extremely Low Frequency Electromagnetic Fields on Melanogenesis through p-ERK and p-SAPK/JNK Pathways in Human Melanocytes. Int. J. Mol. Sci. 2017, 18, 2120. [CrossRef] [PubMed]

20. Kim, Y.M.; Cho, S.E.; Seo, Y.K. The activation of melanogenesis by p-CREB and MITF signaling with extremely low-frequency electromagnetic fields on B16F10 melanoma. Life Sci. 2016, 162, 25-32. [CrossRef] [PubMed]

21. Azuma, N.; Duzgun, S.A.; Ikeda, M.; Kito, H.; Akasaka, N.; Sasajima, T.; Sumpio, B.E. Endothelial cell response to different mechanical forces. J. Vasc. Surg. 2000, 32, 789-794. [CrossRef] [PubMed]

22. Hwang, Y.S.; Kim, Y.J.; Kim, M.O.; Kang, M.; Oh, S.W.; Nho, Y.H.; Park, S.H.; Lee, J. Cannabidiol upregulates melanogenesis through CB1 dependent pathway by activating p38 MAPK and p42/44 MAPK. Chem.-Biol. Interact. 2017, 273, 107-114. [CrossRef] [PubMed]

23. Nishina, A.; Miura, A.; Goto, M.; Terakado, K.; Sato, D.; Kimura, H.; Hirai, Y.; Sato, H.; Phay, N. Mansonone E from Mansonia gagei Inhibited alpha-MSH-Induced Melanogenesis in B16 Cells by Inhibiting CREB Expression and Phosphorylation in the PI3K/Akt Pathway. Biol. Pharm. Bull. 2018, 41, 770-776. [CrossRef] [PubMed]

24. Lee, D.Y.; Lee, J.; Jeong, Y.T.; Byun, G.H.; Kim, J.H. Melanogenesis inhibition activity of floralginsenoside A from Panax ginseng berry. J. Ginseng Res. 2017, 41, 602-607. [CrossRef] [PubMed]

25. Kim, K.N.; Yang, H.M.; Kang, S.M.; Ahn, G.N.; Roh, S.W.; Lee, W.; Kim, D.K.; Jeon, Y.J. Whitening Effect of Octaphlorethol A Isolated from Ishige foliacea in an In Vivo Zebrafish Model. J. Microbiol. Biotechnol. 2015, 25, 448-451. [CrossRef] [PubMed]

26. Choi, T.Y.; Kim, J.H.; Ko, D.H.; Kim, C.H.; Hwang, J.S.; Ahn, S.; Kim, S.Y.; Kim, C.D.; Lee, J.H.; Yoon, T.J. Zebrafish as a new model for phenotype-based screening of melanogenic regulatory compounds. Pigment Cell Res. 2007, 20, 120-127. [CrossRef] [PubMed]

27. Preece, A.W.; Kaune, W.; Grainger, P.; Preece, S.; Golding, J. Magnetic fields from domestic appliances in the UK. Phys. Med. Biol. 1997, 42, 67-76. [CrossRef] [PubMed]

28. D'Angelo, C.; Costantini, E.; Kamal, M.A.; Reale, M. Experimental model for ELF-EMF exposure: Concern for human health. Saudi J. Biol. Sci. 2015, 22, 75-84. [CrossRef] [PubMed]

29. Ma, Q.; Chen, C.; Deng, P.; Zhu, G.; Lin, M.; Zhang, L.; Xu, S.; He, M.; Lu, Y.; Duan, W.; et al. Extremely Low-Frequency Electromagnetic Fields Promote In Vitro Neuronal Differentiation and Neurite Outgrowth of Embryonic Neural Stem Cells via Up-Regulating TRPC1. PLoS ONE 2016, 11, e0150923. [CrossRef] [PubMed]

30. Cheng, Y.; Dai, Y.; Zhu, X.; Xu, H.; Cai, P.; Xia, R.; Mao, L.; Zhao, B.Q.; Fan, W. Extremely low-frequency electromagnetic fields enhance the proliferation and differentiation of neural progenitor cells cultured from ischemic brains. Neuroreport 2015, 26, 896-902. [CrossRef] [PubMed]

31. Destefanis, M.; Viano, M.; Leo, C.; Gervino, G.; Ponzetto, A.; Silvagno, F. Extremely low frequency electromagnetic fields affect proliferation and mitochondrial activity of human cancer cell lines. Int. J. Radiat. Biol. 2015, 91, 964-972. [CrossRef] [PubMed]

32. Podda, M.V.; Leone, L.; Barbati, S.A.; Mastrodonato, A.; Li Puma, D.D.; Piacentini, R.; Grassi, C. Extremely low-frequency electromagnetic fields enhance the survival of newborn neurons in the mouse hippocampus. Eur. J. Neurosci. 2014, 39, 893-903. [CrossRef] [PubMed]

33. Liu, Y.; Liu, W.B.; Liu, K.J.; Ao, L.; Cao, J.; Zhong, J.L.; Liu, J.Y. Overexpression of miR-26b-5p regulates the cell cycle by targeting CCND2 in GC-2 cells under exposure to extremely low frequency electromagnetic fields. Cell Cycle 2016, 15, 357-367. [CrossRef] [PubMed] 
34. Liu, W.-S.; Kuan, Y.-D.; Chiu, K.-H.; Wang, W.-K.; Chang, F.-H.; Liu, C.-H.; Lee, C.-H. The extract of Rhodobacter sphaeroides inhibits melanogenesis through the MEK/ERK signaling pathway. Mar. Drugs 2013, 11, 1899-1908. [CrossRef] [PubMed]

35. Regazzetti, C.; De Donatis, G.M.; Ghorbel, H.H.; Cardot-Leccia, N.; Ambrosetti, D.; Bahadoran, P.; Chignon-Sicard, B.; Lacour, J.P.; Ballotti, R.; Mahns, A.; et al. Endothelial Cells Promote Pigmentation through Endothelin Receptor B Activation. J. Investig. Dermatol. 2015, 135, 3096-3104. [CrossRef] [PubMed]

36. Richardson, J.; Lundegaard, P.R.; Reynolds, N.L.; Dorin, J.R.; Porteous, D.J.; Jackson, I.J.; Patton, E.E. mc1r Pathway regulation of zebrafish melanosome dispersion. Zebrafish 2008, 5, 289-295. [CrossRef] [PubMed]

37. Jin, E.J.; Thibaudeau, G. Effects of lithium on pigmentation in the embryonic zebrafish. Biochim. Biophys. Acta 1999, 1449, 93-99. [CrossRef]

38. Jang, J.Y.; Kim, H.N.; Kim, Y.R.; Choi, W.Y.; Choi, Y.H.; Shin, H.K.; Choi, B.T. Partially purified components of Nardostachys chinensis suppress melanin synthesis through ERK and Akt signaling pathway with cAMP down-regulation in B16F10 cells. J. Ethnopharmacol. 2011, 137, 1207-1214. [CrossRef] [PubMed]

39. Yao, C.; Jin, C.L.; Oh, J.H.; Oh, I.G.; Park, C.H.; Chung, J.H. Ardisia crenata extract stimulates melanogenesis in B16F10 melanoma cells through inhibiting ERK1/2 and Akt activation. Mol. Med. Rep. 2015, 11, 653-657. [CrossRef] [PubMed]

40. Ye, Y.; Wang, H.; Chu, J.H.; Chou, G.X.; Yu, Z.L. Activation of p38 MAPK pathway contributes to the melanogenic property of apigenin in B16 cells. Exp. Dermatol. 2011, 20, 755-757. [CrossRef] [PubMed]

41. Wang, J.; Cui, J.; Zhu, H. Suppression of type I collagen in human scleral fibroblasts treated with extremely low-frequency electromagnetic fields. Mol. Vis. 2013, 19, 885-893. [PubMed]

42. Xu, L.; Xu, Q.H.; Zhou, X.Y.; Yin, L.Y.; Guan, P.P.; Zhang, T.; Liu, J.X. Mechanisms of silver_nanoparticles induced hypopigmentation in embryonic zebrafish. Aquat. Toxicol. 2017, 184, 49-60. [CrossRef] [PubMed]

43. Baek, S.H.; Lee, S.H. Sesamol decreases melanin biosynthesis in melanocyte cells and zebrafish: Possible involvement of MITF via the intracellular cAMP and p38/JNK signalling pathways. Exp. Dermatol. 2015, 24, 761-766. [CrossRef] [PubMed]

44. Chung, S.-Y.; Seo, Y.-K.; Park, J.-M.; Seo, M.-J.; Park, J.-K.; Kim, J.-W.; Park, C.-S. Fermented rice bran downregulates MITF expression and leads to inhibition of $\alpha-\mathrm{MSH}$-induced melanogenesis in B16F1 melanoma. Biosci. Biotechnol. Biochem. 2009, 73, 1704-1710. [CrossRef] [PubMed] 